\title{
REMARKS ON SOUSLIN PROPERTIES AND TREE TOPOLOGIES
}

\author{
WILLIAM G. FLEISSNER ${ }^{1}$
}

\begin{abstract}
We investigate the relation of Souslin (antichain) properties of trees and tree topologies. One result extends a result of Devlin and Shelah by proving, within $Z F C$, the equivalence of four properties for $\omega_{1}$-trees-collectionwise normal, normal and collectionwise Hausdorff, property $\gamma$, and antichain normal and collectionwise Hausdorff. A second result is the construction, assuming $V=L$, of an Aronszajn tree which is not countably metacompact. Third, we show that no tree can be a Dowker space.
\end{abstract}

Certain topological properties of tree spaces can be characterized by Souslin (i.e., antichain) properties of the trees considered as partially ordered sets. An example is the folklore result that the tree topology on an $\omega_{1}$-tree is a Moore space iff the tree is a special Aronszajn tree-i.e., the union of countably many antichains. Devlin and Shelah [DS] obtained characterizations of those trees whose topologies are (a) collectionwise Hausdorff, and (b) (assuming $V=L$ ) normal. This paper continues this line of investigation.

Some properties, such as Moore and collectionwise Hausdorff, can be characterized in $Z F C$-i.e., using only the usual axioms of set theory. Other properties cannot. For example, assuming $M A+\neg C H$, all Aronszajn trees are special and their tree topologies are normal; while assuming $V=L$ the tree topologies of all special Aronszajn trees are not normal. Devlin and Shelah obtained a characterization of normality assuming $V=L$. Although they did not mention it, within their proof are characterizations in $Z F C$ of some other topological properties. We will discuss this in $\S 2$.

The topological properties mentioned above are closely related to the normal Moore space problem. Also related to $\omega_{1}$-trees is the Dowker space problem. In [R], M. E. Rudin "tied together" countably many Souslin trees with the tree topology to get a Dowker space. (We will show in $\$ 4$ that one tree alone cannot be a Dowker space.) It is worth mentioning that a simplification of the proof in that paper yields that the tree topology of a Souslin tree is normal. The topological properties associated with the Dowker space problem are normality, countable paracompactness and countable metacompactness (the last is called property $D$ in [R]). Countable paracompactness behaves similarly to normality with respect to characterization. Assuming $M A+\neg C H$, all Aronszajn trees are special and countably paracompact, while assuming $V=L$ all special Aronszajn trees are not countably paracompact. Countable metacompactness is a much weaker property. It is not

Received by the editors May 19, 1978 and, in revised form, November 19, 1979.

1980 Mathematics Subject Classification. Primary 54F05; Secondary 54D16, 54D18.

${ }^{1}$ Partially supported by NSF grants MCS 78-09484, MCS 79-01848. 
easy to find any example of a regular not countably metacompact space. Within $Z F C$ it can be proven that every special Aronszajn tree is countably metacompact. It is tempting to conjecture that it can be proven within $Z F C$ that every $\omega_{1}$-tree is countably metacompact. In $\$ 3$ we refute this conjecture by assuming $V=L$, specifically $\diamond^{+}$, to construct an Aronszajn tree which is not countably metacompact.

1. Notation and convention. An ordinal is the set of its predecessors. Cardinals are identified with initial ordinals. The cardinality of a set $X$ is denoted $|X|$. A subset $C$ of $\omega_{1}$ is club (closed and unbounded) iff $|C|=\omega_{1}$ and whenever $D$ is a countable subset of $C$, then $\sup D \in C$.

A tree $\mathcal{T}$ is a partially ordered set $\left(T,<_{T}\right)$ such that for every $t \in T$, the set $\hat{t}=\{s \in T: s<t\}$ is well ordered by $<_{T}$. The order type of $\left(\hat{t},<_{T}\right)$ is denoted $\operatorname{ht}(t) ;\{t \in T: \operatorname{ht}(t)=\alpha\}$ is denoted by $T_{\alpha}$. A branch of $\mathcal{T}$ is a maximal totally ordered subset of $T$; a branch $b$ is an $\alpha$-branch if the order type of $\left(b,<_{T}\right)$ is $\alpha$. For $C$ a set of ordinals, $T-T \uparrow C=\{t \in T$ : ht $(t) \notin C\}$. An antichain of $T$ is a pairwise incomparable subset of $\mathcal{T}$.

$\mathcal{T}$ is an $\omega_{1}$-tree iff (i) $\{h t(t): t \in T\}=\omega_{1}$, (ii) for all $\alpha<\omega_{1},\left|T_{\alpha}\right|=\omega$, (iii) for every $t \in T$ and for every $\alpha, \operatorname{ht}(t)<\alpha<\omega_{1}, t$ has at least two successors of height $\alpha$, and (iv) if $h t(t)=h t(s)$ is a limit ordinal, $t=s$ iff $\hat{t}=\hat{s}$. An Aronszajn tree is an $\omega_{1}$-tree with no $\omega_{1}$-branch; a Souslin tree is an $\omega_{1}$-tree with no uncountable antichain.

Let $\mathcal{T}$ be an $\omega_{1}$-tree. The tree topology on $\mathcal{T}$ has a basis of all sets of the following forms:

$$
\begin{array}{ll}
{[t, s)=\{u \in T: t \leqslant u<s\}} & \text { where } t \in T_{0}, s \in T, \\
(t, s)=\{u \in T: t<u<s\} & \text { where } t \in T, s \in T .
\end{array}
$$

When we say that $\mathcal{T}$ is normal, we mean the tree topology on it is normal (not that $\mathcal{T}$ is a normal $\alpha$-tree as defined in [J, p. 218]). A topological space is collectionwise Hausdorff iff every closed discrete collection of points can be simultaneously separated by disjoint open sets; a topological space is collectionwise normal iff every closed discrete collection of closed sets can be simultaneously separated by disjoint open sets.

An $\omega_{1}$-tree $\mathcal{T}$ is said to have property $\gamma$ [DS] iff whenever $A$ is an antichain of $\mathcal{T}$ there is a club subset $C$ of $\omega_{1}$ such that $T-T \uparrow C$ contains a closed neighborhood of $A$. An $\omega_{1}$-tree $\mathcal{T}$ is said to be almost Souslin iff whenever $A$ is an antichain of $\mathcal{T}$ then $\{\operatorname{ht}(a): a \in A\}$ is not stationary. Clearly property $\gamma$ implies almost Souslin; it is not hard to show (see [DS, Theorem 3.3]) that $\mathcal{T}$ is almost Souslin iff the tree topology on $\mathcal{T}$ is collectionwise Hausdorff.

The tree topology on an $\omega_{1}$-tree $\mathcal{T}$ is said to be antichain normal iff whenever $H$ and $K$ are disjoint closed subsets of $T$ then they can be separated by disjoint open sets if at least one of $H$ and $K$ is an antichain.

A space is countably paracompact iff every countable open cover has a locally finite refinement. A space is countably metacompact iff every countable open cover has a point finite open refinement. 
2. Collectionwise normality and property $\gamma$. In this section we prove within $Z F C$ that four properties of an $\omega_{1}$-tree are equivalent. The hardest part-(d) $\rightarrow$ (a)-is essentially Theorem 4.1 of [DS], but we have strengthened the conclusion and used topological ideas to clean up some details. We derive the characterization of normal tree topologies assuming $V=L$ as a corollary of Theorems 2.1 and 2.2. (The key part of Theorem 4.2 of [DS] is to reprove a special case of Theorem 2.2.)

THEOREM 2.1. The following are equivalent for an $\omega_{1}$-tree $\mathcal{T}$.

(a) The tree topology is collectionwise normal.

(b) The tree topology is normal and collectionwise Hausdorff.

(c) $\mathcal{T}$ has property $\gamma$.

(d) The tree topology is antichain normal and collectionwise Hausdorff.

TheORem 2.2 [F]. Assuming $V=L$, every normal $T_{2}$ space of character $<2^{\omega}$ is collectionwise Hausdorff. In particular, every normal $\omega_{1}$-tree is collectionwise Hausdorff.

COROllaRY 2.3 [DS]. Assuming $V=L$, an $\omega_{1}$-tree $\mathcal{T}$ has property $\gamma$ iff its tree topology is normal.

Proof of Theorem 2.1. (a) $\rightarrow$ (b) $\rightarrow$ (d) is obvious.

(b) $\rightarrow$ (c). Because $\mathcal{T}$ is collectionwise Hausdorff, if $A$ is an antichain of $\mathcal{T}$, then $A^{*}=\{\operatorname{ht}(a): a \in A\}$ is not stationary. Let $C$ be a club set disjoint from $A^{*}$. Apply normality to the disjoint closed sets $A$ and $\{t \in T: \operatorname{ht}(t) \in C\}$.

(c) $\rightarrow$ (d). Let $A$ be an antichain of $\mathcal{T}$ and $H$ a closed set disjoint from $A$. Because $\mathcal{T}$ has property $\gamma, A^{*}=\{\operatorname{ht}(a): a \in A\}$ is not stationary, so let $C$ be a club set disjoint from $A^{*}$. From property $\gamma$ we obtain disjoint open sets $U, V$ satisfying

$$
U \supset A, \quad V \supset\{t \in T: \operatorname{ht}(t) \in C\} .
$$

Next notice that $T-T \uparrow C$ is an open metrizable subset, so we can find disjoint open subsets $U^{\prime}, V^{\prime}$ satisfying $U^{\prime} \supset A, V^{\prime} \supset H \cap(T-T \uparrow C)$. Then $U \cap U^{\prime}$ and $V \cup V^{\prime}$ are disjoint open subsets separating $A$ and $H$.

(d) $\rightarrow$ (a). Let $\mathcal{Y}=\left\{Y_{i}: i \in I\right\}$ be a discrete family of closed sets. We define antichains $A_{n}, n \in \omega$, by induction on $n$. Let $A_{0}$ be the set of $<_{T}$-minimal elements of $\cup \mathcal{Y}$. For $a \in A_{n}$, let

$$
T^{(a)}=\left\{t \in T: a<_{T} t\right\},
$$

and let $i(a) \in I$ be such that $a \in Y_{i(a)}$. Let $B(a)$ be the set of $T$-minimal elements of $T^{(a)} \cap\left(\cup \mathcal{Y}-Y_{i(a)}\right)$. Set

$$
A_{n+1}=\bigcup\left\{B(a): a \in A_{n}\right\} .
$$

Note that for every $t \in T, \hat{t} \cap\left(\cup_{n \in \omega} A_{n}\right)$ is finite. For if it were infinite, it would have a limit point. That limit point would contradict the assumption that $\mathcal{Y}$ is discrete.

For $a \in A_{n}$, set

$$
X(a)=T^{(a)}-\bigcup\left\{T^{(b)}: b \in B(a)\right\}
$$


By the previous paragraph

$$
\mathscr{X}=\left\{X(a): a \in A_{n}, n \in \omega\right\} \cup\left\{\left\{t \in T: \hat{t} \cap A_{0}=\varnothing\right\}\right\}
$$

is a partition of $T$ into closed and open subsets. So it is sufficient to separate $\mathcal{Y} \mid X=\left\{Y_{i} \cap X: i \in I\right\}$ for each $X \in \mathcal{X}$. Let $X \in \mathcal{X}$ be $X(a)$ (the other case is simpler). Use antichain normal to separate $Y_{i(a)} \cap X$ from $B(a)$. Then use collectionwise Hausdorff to separate the points of the closed discrete set $B(a)$.

3. Countable metacompactness and $\diamond^{+}$. In this section we use $\diamond^{+}$, a consequence of $V=L$, to construct an Aronszajn tree which is not countably metacompact. Our goal is to define a tree $\mathcal{T}$ with antichain $A$ and partition $A$ into $\left\{A_{n}, n \in \omega\right\}$. To show $\mathcal{T}$ is not countably metacompact we will set $U_{n}=(T-A) \cup A_{n}$. Clearly $\mathscr{U}=\left\{U_{n}: n \in \omega\right\}$ is a countable open cover of $\mathcal{T}$. Suppose there were a pointfinite open refinement $R$ of $\mathcal{Q}$. Define, for $r \in R, n(R)$ to be the least $n$ such that $R \subset U_{n}$. Define for $t \in T f(t)=\sup \{n(R): t \in R \in \Re\}$. Then $f$ would satisfy

(i) $f: T \rightarrow \omega$,

(ii) if $a \in A_{n}$, then $f(a)=n$,

(iii) for all $t \in T-T_{0}$ there is $s \in t$ such that for all $u \in(s, t), f(u)>f(t)$.

We will use $\diamond^{+}$in constructing $\mathcal{T}, A$ so that no such $f$ exists. The key idea in preventing such $f$ is the following observation, whose routine proof is omitted.

LEMMA 3.1. Let $b$ be an $\omega_{1}$-branch of $\mathcal{T}$, and $f: b \rightarrow \omega$. Let $n$ be the least natural number such that $f^{\leftarrow}\{n\}$ is uncountable. Set $C_{b}=\left\{\gamma \in \omega_{1}: f^{\leftarrow}\{n\} \cap \gamma\right.$ is cofinal in $\left.\gamma, \gamma>\sup f^{\leftarrow}\{0,1, \ldots, n-1\}\right\}$. Then $C_{b}$ is a club set.

Our plan is inductively define $<^{\alpha}=<_{T} \cap\left(\cup_{\beta<\alpha} T_{\beta} \times \cup_{\beta<\alpha} T_{\beta}\right)$. At each stage, $\diamond^{+}$will give us countably many $f \uparrow \alpha$ 's to look at. If $f \uparrow \alpha$ already does not satisfy (ii), (iii) above, there is nothing that needs to be done. If $f \uparrow \alpha$ satisfies (ii) and (iii) but we can prevent any extension of $f \uparrow \alpha$ satisfying (ii), (iii), we do so. Otherwise we continue growing a branch associated with $f \uparrow \alpha$. Lemma 3.1 applied to the branch associated with the initial segments of a function $f: T \rightarrow \omega$ guarantees that at some stage we can prevent $f$ from satisfying (ii) and (iii).

The construction sketched above requires $\diamond^{+}$and not merely $\diamond$ because in the otherwise case we need to be sure that the branch associated with $f \uparrow \alpha$ has been continued up to level $\alpha$. We proceed to the technical details.

$\diamond^{+}$is the assertion that there is a sequence $\left\{W_{\alpha}: \alpha<\omega_{1}\right\}$ satisfying

(a) for each $\alpha<\omega_{1}, W_{\alpha}$ is a countable family of subsets of $\alpha$,

(b) for each $X \subset \omega_{1}$, there is a set $C_{X}$ club in $\omega_{1}$ such that for all $\gamma \in C_{X}$, $X \cap \gamma \in W_{\gamma}$ and $C_{X} \cap \gamma \in W_{\gamma}$.

We will add the harmless additional condition

(c) $W_{\alpha} \neq \varnothing$ implies $\alpha$ is a limit ordinal.

We will find it useful to collect pairs of elements of $W$ which might be $X \cap \gamma$ and $C_{X} \cap \gamma$. For $\alpha<\omega_{1}$ let $Z_{\alpha}$ be the set of pairs $(x, c)$ satisfying

(d) $x \in W_{\alpha}, c \in W_{\alpha}$,

(e) $c$ is club in $\alpha$,

(f) for all $\gamma \in c, x \cap \gamma \in W_{\gamma}$ and $c \cap \gamma \in W_{\gamma}$. 
We will use the $W$ sequence to consider branches through the tree, and functions from the tree to $\omega$. Such objects are not subsets of $\omega_{1}$, but they can be coded as subsets of $\omega_{1}$. To accomplish this coding, let $\theta$ be a bijection from $\omega_{1}$ to $\omega_{1} \times \omega \times \omega$ such that for each limit ordinal $\lambda<\omega_{1}, \theta \uparrow \lambda$ is a bijection from $\lambda$ to $\lambda \times \omega \times \omega$. Let $\rho$ be a similar bijection from $\omega_{1}$ to $\omega_{1} \times \omega$.

We will define $<^{\alpha}, A_{n}^{\alpha}$ (for all $n \in \omega$ ), and $B^{\alpha}$ by induction on $\alpha<\omega_{1}$. The idea is that we will set $<=\cup_{\alpha<\omega_{1}}<^{\alpha}, A_{n}=\cup_{\alpha<\omega_{1}} A_{n}^{\alpha}$ and $B=\cup_{\alpha<\omega_{1}} B^{\alpha}$. Then $\mathcal{T}=\left(\left(\omega_{1} \times \omega\right),<\right)$ will be a tree, $A=\cup_{n \in \omega} A_{n}$ an antichain of $\mathcal{T}$, and $B$ will be a function from (codes of) initial segments of functions $f$ from $T$ to $\omega . B$ associates a branch with functions $f$ which might satisfy (ii) and (iii).

For notational convenience, we have made the elements of $A$ maximal in $\mathcal{T}$. We will later "graft" an Aronszajn tree above each element of $A$ to make an $\omega_{1}$-tree.

The branch associated with $f$ is the set of all predecessors of elements of the form $\boldsymbol{B}\left(\left((\theta \leftarrow f) \cap \gamma, C_{\theta \leftarrow f} \cap \gamma\right)\right)$, where defined. Hypothesis 9 insures that that set is indeed a branch and that different branches are associated with different functions. Hypothesis 11, when applicable, prevents $f$ from satisfying (i), (ii), (iii). After Hypothesis 11 is applied, Hypothesis $10(\mathrm{c})$ says that we no longer continue to grow the branch associated with $f$.

We now define $<^{\alpha}, A_{n}^{\alpha}$ (for $n \in \omega$ ) and $B^{\alpha}$ by induction on $\alpha<\omega_{1}$ to satisfy the following hypotheses.

1. For all $\beta<\alpha$ and $n \in \omega,<^{\beta} \subset<^{\alpha}, A_{n}^{\beta} \subset A_{n}^{\alpha}$, and $B^{\beta} \subset B^{\alpha}$.

2. $\mathfrak{T}^{\alpha}=\left((\alpha \times \omega),<^{\alpha}\right)$ is a tree of height $\alpha$.

3. For all $(\beta, n) \in \alpha \times \omega, \operatorname{ht}((\beta, n))=\beta$.

4. $\left\{A_{n}^{\alpha}: n \in \omega\right\}$ is a disjoint family and $A^{\alpha}=\cup_{n \in \omega} A_{n}^{\alpha}$ is an antichain of $\mathscr{T}^{\alpha}$.

5. If $t \in A^{\alpha}$ then $t$ is a maximal element of $\tilde{T}^{\alpha}$.

6. If $t \in T^{\alpha}-A^{\alpha}$, then for all $\beta, \operatorname{ht}(t)<\beta<\alpha$, there are distinct $s, s^{\prime} \in T_{\beta}^{\alpha}$ such that $t<{ }^{\alpha} s^{\prime}$ and $t<{ }^{\alpha} s,\left(t, s^{\prime}\right] \cap$ range $B^{\alpha}=\varnothing=\left(t, s^{\prime}\right] \cap$ range $B^{\alpha}$.

7. $a \in A^{\alpha}$ implies that $a \in$ range $B^{\alpha}$ and that ht $(a)$ is a limit ordinal.

8. $B^{\alpha}$ is a function, $\operatorname{dom} B^{\alpha} \subset \cup_{\beta<\alpha} Z_{\beta}$ and $(x, c) \in Z_{\beta} \cap \operatorname{dom} B$ implies $\operatorname{ht}\left(B^{\alpha}((x, c))\right)=\beta$.

9. If $(x, c) \in \operatorname{dom} B^{\alpha}$ and $(y, d) \in \operatorname{dom} B^{\alpha}$ then $B^{\alpha}((y, d))<^{\alpha} B^{\alpha} B^{\alpha}((x, c))$ iff there is $\gamma \in c$ such that $(y, d)=(x \cap \gamma, c \cap \gamma)$.

10. For all $\beta<\alpha$ and $(x, c) \in Z_{\beta},(x, c) \in \operatorname{dom} B^{\alpha}$ iff

(a) $\theta \rightarrow x$ is a function from $T^{\beta}$ to $\omega$.

(b) $a \in A_{n}^{\beta}$ implies $\theta \rightarrow x(a)=n$.

(c) For all $t \in T^{\beta}-T_{0}^{\beta}$ there is $s<^{\alpha} t$ such that for all $u \in(s, t], \theta \rightarrow x(u) \geqslant$ $\theta \rightarrow x(t)$.

11. If $(x, c) \in \operatorname{dom} B^{\alpha}$, then $t=B^{\alpha}((x, c)) \in A_{n}^{\alpha}$ if $n$ is the least natural number such that $(\theta \rightarrow x) \rightarrow\{n\}$ is cofinal in $\hat{t}$.

12. For all $\beta<\alpha$ and $y \in W_{\beta}$ if $\rho \rightarrow y$ is a $\beta$-branch of $T^{\beta}$ and range $B^{\beta}$ is not cofinal in $\rho \rightarrow y$, then there is no $t \in T_{\beta}^{\alpha}$ such that $\rho \rightarrow y+\hat{t}$.

The above induction can be easily carried out. Set $<^{0}=A_{n}^{0}$ (for all $\left.n \in \omega\right)=B^{0}$ $=\varnothing$. If $\lambda$ is a limit ordinal set $<^{\lambda}=\cup_{\alpha<\lambda}<^{\alpha}, B^{\lambda}=\cup_{\alpha<\lambda} B^{\alpha}$, and for all $n \in \omega, A_{n}^{\lambda}=\cup_{\alpha<\lambda} A_{n}^{\alpha}$. 
If $\alpha=\delta+1$, where $\delta=\nu+1$, define $<^{\alpha}$ so that every element of $T_{\nu}^{\delta}-A_{\nu}$ has two immediate successors in $T_{\delta}^{\alpha}$.

Finally, suppose $\alpha=\lambda+1$ where $\lambda$ is a limit ordinal. Assign the elements of $T_{\lambda}^{\alpha}$ in the following order. First, define $B^{\alpha}$ to satisfy Hypotheses 1, 9, 10. It is Hypothesis 9 which requires $\diamond^{+}$rather than $\diamond$. We must define $B^{\lambda}((x, c))$ for certain $(x, c) \in Z_{\lambda}$. Because $c$ is closed in $\lambda$ there are three possibilities: $c$ is empty, $c$ has a last element, or $c$ is cofinal in $\lambda$. In each of these cases, there is no problem in defining $B^{\lambda}((x, c))$ to satisfy Hypothesis 9 . The point is that the case $c$ has no last element and $c$ is not cofinal in $\lambda$ does not occur. Second, define $A_{n}^{\alpha}$ (for $n \in \omega$ ) to satisfy Hypotheses 1, 7, 11. Third, assign elements to satisfy Hypotheses 6, 12. Everything can be done in one step except satisfying Hypothesis 6. Given $t \in T_{\beta}^{\lambda}$ $-A^{\lambda}$, we must extend $\hat{t} \cup\{t\}$ to be a $\lambda$-branch with elements above $t$ not in range $B^{\alpha}$ and the $\lambda$-branch not $\rho(y)$ for some $y \in W_{\lambda}$. There are countably many things to avoid, and the confinality of $\lambda$ is $\omega$, so we avoid the countably many things to be avoided one by one, using the induction hypothesis that Hypothesis 6 is satisfied for $\lambda$.

We finish defining the example by grafting an Aronszajn tree above each element of $A$.

Having defined $\mathcal{T}$, we must verify that it is not countably metacompact. Aiming for a contradiction, we suppose not. We argue as at the beginning of this section, using $A=\cup_{n \in \omega} A_{n}$ to define a countable open cover, and using the hypothesized refinement to get a function $f$ satisfying (i), (ii) and (iii). Because $\theta$ is a bijection, $f$ will be $\theta \rightarrow X$ for some $X \subset \omega_{1}$. From $\diamond^{+}$we get a club set $C$ such that $\gamma \in C$ implies that $(X \cap \gamma, C \cap \gamma) \in Z_{\gamma}$. Because $f$ satisfies (i), (ii) and (iii), induction Hypothesis 10 yields that for all $\gamma \in C,(X \cap \gamma, C \cap \gamma) \in \operatorname{dom} B$. Then induction Hypotheses 8, 9 yields that $b=\{t \in T:(\exists \gamma \in C)(t<B(x \cap \gamma, C \cap \gamma))\}$ is an $\omega_{1}$-branch. We apply Lemma 3.1 to get another club set $C_{b}$. Let $\gamma \in C \cap C_{b}$. Since $\gamma \in C_{b}$, there is (a least) $n \in \omega$ such that $\left\{\alpha<\gamma: f\left(b_{\alpha}\right)<n\right\}$ is cofinal in $\gamma$. Because $\gamma \in C,(x \cap \gamma, c \cap \gamma) \in \operatorname{dom} B$ and by induction Hypothesis $11, b_{\gamma} \in$ $A_{n+1}$. Since $f$ satisfies (ii), $f\left(b_{\gamma}\right)=n+1$. Since $f$ satisfies (iii) $\left\{\alpha<\gamma: f\left(b_{\alpha}\right)<n\right\}$ is not cofinal in $\gamma$. Contradiction.

A similar but simpler "usual $\diamond$ argument" using induction Hypothesis 12, shows that there is no $\omega_{1}$-branch through $\mathcal{T}$. The attentive reader will note that if we continue each branch associated with a function to an $\omega_{1}$-branch (e.g., we delete Hypotheses 5, 10(c), 12 and replace " $t \in T^{\alpha}-A^{\alpha "}$ with " $t \in T^{\alpha}$ ") the above construction yields a not countably metacompact $\omega_{1}$-tree with $2^{\omega_{1}} \omega_{1}$-branches.

4. There are no Dowker trees. Peter Nyikos has modified an idea of a preliminary draft of this paper to give the following result. He has graciously suggested that it be included in this paper.

THEOREM 4.1. If a tree $\mathcal{T}$ is either antichain normal or collectionwise Hausdorff, then $\mathcal{T}$ is countably metacompact.

COROLlaRy 4.2. There are no Dowker trees. 
Proof of Theorem 4.1. Let $\mathscr{U}=\left\{U_{n}: n \in \omega\right\}$ be a countable open cover of the tree $T$. For each $n \in \omega$, define

$$
V_{n}=\left\{t \in T:\{t\} \cup \hat{t} \subseteq \bigcup_{m<n} U_{n}\right\} .
$$

Then $\cup_{n<\omega} V_{n}=T, V_{n} \subset \cup_{m<n} U_{n}$, and (closure $V_{n}$ ) $-V_{n}$ is an antichain. Define $W_{0}=V_{0}, W_{n+1}=V_{n+1}-\left(\right.$ closure $\left.V_{n}\right)$. Then $\mathscr{W}=\left\{U_{m} \cap W_{n}: m<n<\right.$ $\omega\}$ is point-finite, open, refines $\mathcal{Q}$, and covers all of $T$ except the closed discrete subset $Y=\cup_{n<\omega}\left(\left(\right.\right.$ closure $\left.\left.V_{n}\right)-V_{n}\right)$. Set $A_{n}=Y \cap\left(U_{n}-\cup_{m<n} U_{m}\right)$; by either antichain normal or collectionwise Hausdorff, there is a disjoint open family $\mathcal{G}=\left\{G_{n}: n \in \omega\right\}$ such that for each $n \in \omega, A_{n} \subset G_{n} \subset U_{n}$. Then $\mathscr{Q} \cup \mathcal{G}$ is a point finite open refinement of $\mathcal{U}$.

4.3. Question. Suppose the tree topology on $\mathcal{T}$ is such that every closed subset of $T$ is $G_{\delta}$. Must $T$ be the union of countably many antichains? Equivalently, does perfect imply Moore for tree spaces?

\section{BIBLIOGRAPHY}

[DS] K. J. Devlin and S. Shelah, Souslin properties and tree topologies, Proc. London Math. Soc. 39 (1979), 237-252.

[F] W. G. Fleissner, Normal Moore spaces in the constructible universe, Proc. Amer. Math. Soc. 46 (1974), 294-298.

[R] M. E. Rudin, Countable paracompactness and Souslin's problem, Canad. J. Math. 7 (1955), 543-547.

[J] T. Jech, Set theory, Academic Press, New York, 1978.

Department of Mathematics, University of Pittsburgh, Pittsburgh, Pennsylvania 15260 